\title{
ANÁLISE DA INFLUÊNCIA DOS PARÂMETROS DE LAMINAÇÃO A QUENTE NAS PROPRIEDADES MECÂNICAS E MICROESTRUTURA E DO VERGALHÃO CA50*
}

\author{
Rafael Lopes ${ }^{1}$ \\ Gláucio Soares da Fonsceca² \\ Viviane de Melo Borges ${ }^{3}$ \\ Ilaciara Medeiros de Freitas ${ }^{4}$
}

\section{Resumo}

Os aços utilizados na fabricação do vergalhão CA50 conforme norma NBR7480 são usados em todo o Brasil. A grande aplicação deste tipo de produto é na indústria de construção civil em função de sua excelente resistência mecânica e soldabilidade. Estas características permitem o avanço na construção de grandes empreendimentos. Em 2009, a Votorantim Siderurgia na sua Unidade de Resende, iniciou a produção do vergalhão CA50 em escala, procurando aumentar a sua participação no mercado de construção civil. Foi identificada uma grande variação nos resultados de limite de escoamento nos ensaios de liberação de produto realizados a partir de amostras coletadas no início das bobinas de vergalhão CA50. Em função disso, foi feita a caracterização do limite de escoamento e microestrutura ao longo da bobina onde foi identificado que esta grande variação do limite de escoamento estava presente em toda a bobina. Desta forma, foram definidos os principais parâmetros do processo de laminação que poderiam estar influenciando na variação do limite de escoamento e realizado um planejamento de experimentos (DOE) para o vergalhão CA50 $16,0 \mathrm{~mm}$, aço 1030, onde foram identificadas que a combinação da melhor distribuição da vazão de água nas Caixas d'água utilizadas no tratamento térmico de têmpera e auto revenido, somada à maior distribuição das espiras no pelo Formador e à ventilação do Stelmor no mínimo apresentaram resultados de limite de escoamento acima do mínimo especificado pela norma NBR $7480 \mathrm{com}$ menor desvio padrão quando comparado aos parâmetros de processo que eram utilizados na produção do vergalhão CA50.

Palavras-chave: Vergalhão CA50; Propriedades Mecânicas; Microestrutura; DOE.

\section{ANALYSIS OF THE INFLUENCE OF ROLLING MILL PARAMETERS IN MICROSTRUCTURE AND MECHANICAL PROPERTIES OF RIBBED REBAR}

\begin{abstract}
The steels used in the manufacture of rebar CA50 according to NBR7480 are used throughout Brazil. The great application of this type of product is in the civil construction industry due to its excellent mechanical strength and weldability. These characteristics allow the advance in the construction of large enterprises. In 2009, Votorantim Siderurgia at its Resende Unit started producing CA50 rebar in scale, seeking to increase its participation in the civil construction market. A large variation in the yield limit results was identified in the product release tests performed from samples collected at the beginning of the CA50 rebar coils. Due to this, the yield strength and microstructure were characterized along the coil where it was identified that this large variation of the yield strength was present throughout the coil. In this way, the main parameters of the rolling process that could be influencing the variation of the yield strength were determined, and an Design of Experiment (DOE) for the CA50 16.0mm rebar, steel 1030 was performed, where it was identified that the combination of the best distribution of the water flow in the Water Boxes used in the heat treatment of quenching and self tempering, added to the greater distribution of the rings in the Laying Head and to the ventilation of the Stelmor at minimum presented results of yield strength above the minimum specified by norm NBR 7480 with lower standard deviation when compared to process parameters that were used in the production of CA50 rebar.
\end{abstract}

Keywords: CA50 Rebar; Mechanical properties; Microstructure; DOE.

Engenheiro Metalurgista UFF, Mestrando em Engenharia Metalúrgica UFF, Engenheiro de Processo, Votorantim Siderurgia, Resende, Rio de Janeiro, Brasil. 
2 Doutor em Engenharia Metalúrgica, Departamento Engenharia Mecânica UFF, Programa de Pósgraduação em Engenharia Metalúrgica, PPGEM, UFF.

3 Engenheira Química, Mestranda em Engenharia Metalúrgica UFF, Engenheira de Qualidade, Gestão e Qualidade, Votorantim Siderurgia/Universidade Federal Fluminense, Resende, Rio de Janeiro - Brasil.

4 Engenheira Metalurgista UFF, Engenheira de Qualidade, Gestão e Qualidade, Votorantim Siderurgia/Universidade Federal Fluminense, Resende, Rio de Janeiro - Brasil. 


\section{INTRODUÇÃO}

O mercado siderúrgico tem cada vez exigido produtos com alta resistência e ductilidade. A fim de obter alta resistência ao escoamento na laminação a quente, é necessário a adição de elementos de liga. No entanto, estas adições reduzem a soldabilidade e a ductilidade. Além disso, o custo de fabricação do aço também aumenta, fato não desejado num mercado tão competitivo como atualmente.

$O$ investimento no desenvolvimento de novas tecnologias tem-se tornado cada vez mais evidente, visto que se tem uma busca contínua na qualidade diferenciada dos produtos e serviços oferecidos, com um baixo custo de produção (Sung, 2014). Desta forma, para obter um produto com alto limite de escoamento e boa ductilidade e soldabilidade, a partir de um aço com baixo teor de carbono e outros elementos de liga de forma economicamente viável, o processo de tratamento térmico de tempera e auto revenido após o último passe de laminação é uma solução apropriada.

No processo de tempera e auto revenido um intenso resfriamento com água é aplicado na superfície do material após o último passe de laminação, de forma que a superfície é temperada. O resfriamento é interrompido antes que o núcleo seja atingido (Official Publications of the European Communities, 1992). Desta forma, o núcleo permanece mais aquecido quando comparado à superfície. Este gradiente de temperatura permite que o calor seja dissipado do núcleo para a superfície causando o revenimento da martensita. O núcleo permanece com austenita. $\mathrm{Na}$ esteira de resfriamento controlado, através da ventilação forçada com ar, a estrutura do núcleo com austenita é transformada em ferrita e perlita. Portanto, a estrutura final consiste em numa ótima combinação de uma resistente camada de martensita revenida com núcleo dúctil de ferrita e perlita (Viventsov et. al, 2010).

Figura 1 - Seção transversal de uma barra tratada termicamente através do controle do sistema METCS@. (Copyright (C) Morgan Construction Company Manual Morgan

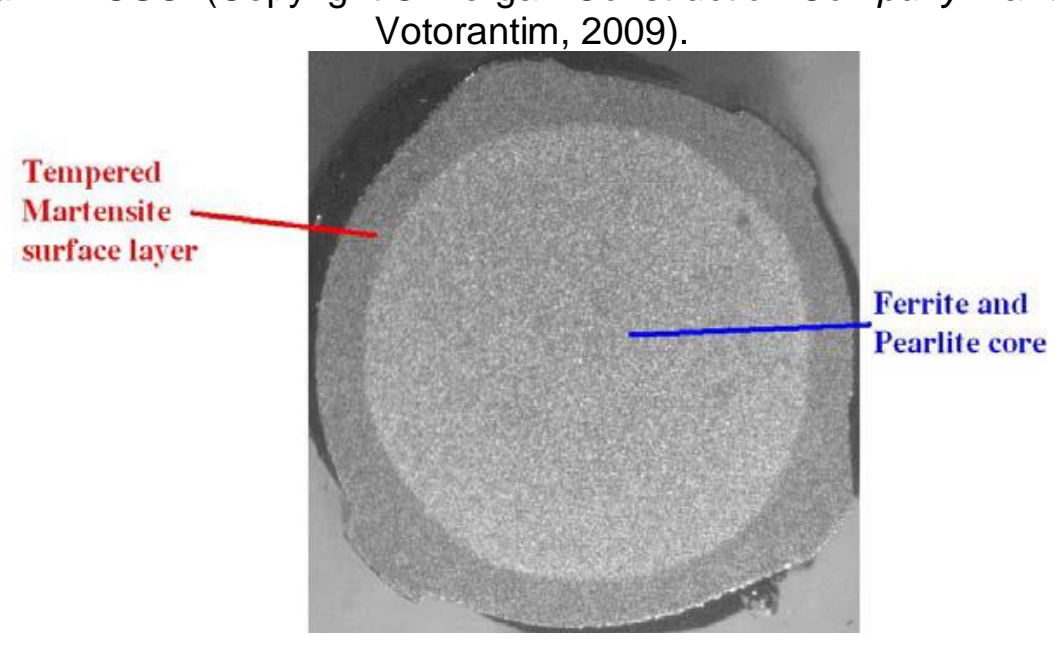

Materiais que são submetidos ao processo de têmpera e auto revenido, seguido de resfriamento com ar forçado apresentam os melhores valores de limite de escoamento, com um razoável alongamento quando comparado a outros meios de resfriamentos utilizados em aços ligados com vanádio e nióbio (Zaky et. al., 2008). Em alguns casos, de acordo com os parâmetros de resfriamento controlado, a austenita logo abaixo da camada temperada é transformada parcialmente ou completamente em bainita. Como resultado, três camadas concêntricas são 
formadas na seção transversal do vergalhão: uma resistente e tenaz camada de martensita revenida na superfície, uma camada intermediária de bainita e martensita e, um núcleo com estrutura refinada e tenaz ferrita e perlita (Souvik et. al., 2014).

O sistema de tratamento térmico é composto por 5 caixas d'água que têm a função de uniformizar o resfriamento a barra à medida que a mesma passa pela caixa, além disso, retardam o crescimento do grão garantindo a microestrutura final desejada. O tempo de resfriamento é em função da redução de temperatura desejada, da velocidade de laminação, da temperatura da água de contato e da eficiência de resfriamento das caixas. O resfriamento após o bloco é dividido, conforme figura 2, em duas zonas, 2 e 3, cada uma contendo 02 caixas. A vazão da zona 2 é ajustada de modo manual, enquanto que a da zona 3 é automática em função da temperatura do pirômetro do Formador de Espiras.

Figura 2 - Esquema dos equipamentos do tratamento térmico de têmpera e auto revenido do vergalhão CA50 da Votorantim Siderurgia (arquivo pessoal, 2014).

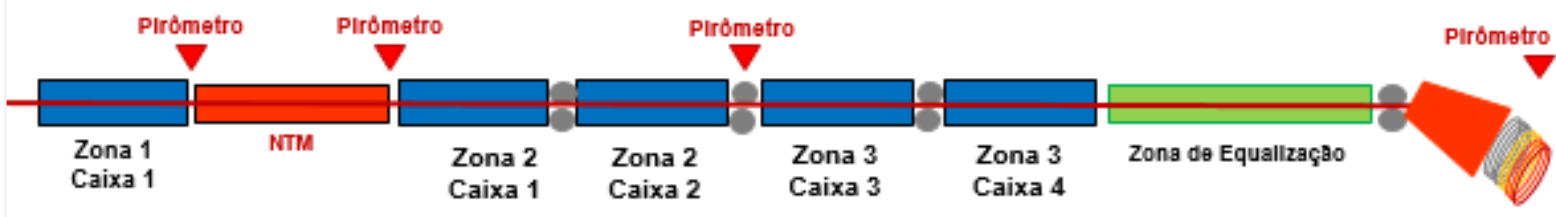

Após passar pelas caixas d'água o material é submetido ao resfriamento com ar no Stelmor, por meio de 6 ventiladores, como mostrado na figura 3 a seguir.

Figura 3 - Desenho esquemático com visão lateral do STELMOR ${ }^{\circledR}$.

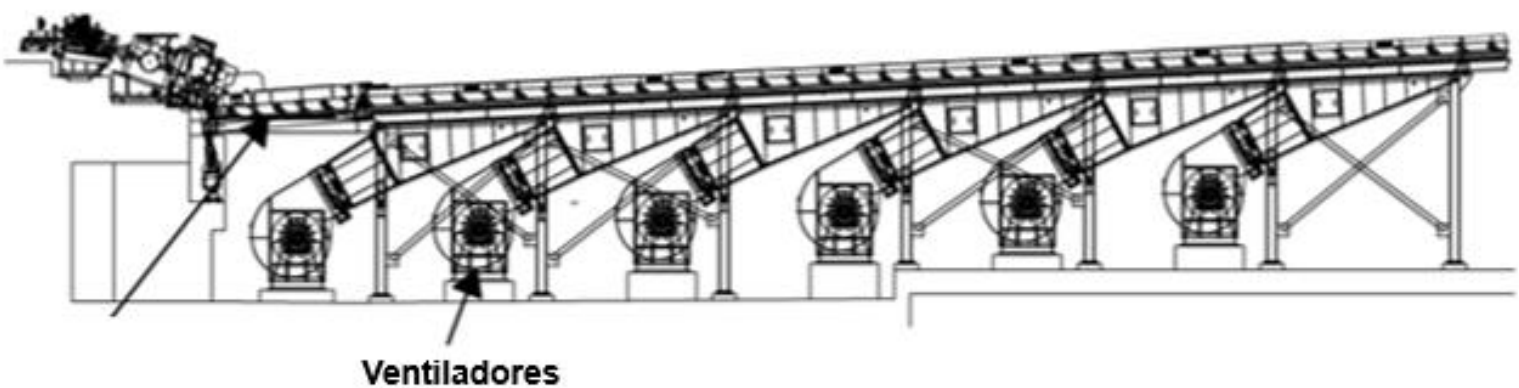

O entendimento das variáveis que afetam as propriedades mecânicas e microestrutura é necessário para otimizar os parâmetros de processo e garantir a produção do vergalhão com baixo custo e com características que atendam à norma.

\section{MATERIAIS E MÉTODOS}

O vergalhão CA50 16,0 mm 1030 utilizado nesse trabalho foi produzido na laminação a quente da Votorantim Siderurgia Unidade Resende, Rio de Janeiro. Este laminador é composto de 01 Forno de Reaquecimento, 28 gaiolas de laminação, 05 caixas de água responsáveis pelo controle de temperatura e tratamento térmico de têmpera e auto revenido. A composição química especificada para ovaço 1030 mostrada na Tabela 1. 
Tabela 1 - Composição Química especificada para o aço 1030.

\begin{tabular}{llccccccc}
\hline & & $\mathrm{C}(\%)$ & $\mathrm{Mn}(\%)$ & $\mathrm{Si}(\%)$ & $\mathrm{P}(\%)$ & $\mathrm{S}(\%)$ & $\begin{array}{c}\text { Mo } \\
(\%)\end{array}$ & $\begin{array}{c}\mathrm{N} \\
(\mathrm{ppm})\end{array}$ \\
\hline \multirow{2}{*}{$\mathbf{1 0 3 0}$} & Lim. Sup. & 0,28 & 0,60 & 0,15 & & & & - \\
& Lim. Inf. & 0,34 & 0,90 & 0,30 & 0,05 & 0,04 & 0,10 & 150,00 \\
\hline
\end{tabular}

Para definição do problema foram produzidas 45 bobinas de CA50 16,0 mm com os parâmetros de processo padrões onde uma bobina foi selecionada aleatoriamente para coleta das amostras. Foi retirada a $5^{\mathrm{a}}, 8^{\mathrm{a}}, 10^{\mathrm{a}}, 12^{\mathrm{a}}, 15^{\mathrm{a}}, 18^{\mathrm{a}}, 20^{\mathrm{a}}$, $25^{\mathrm{a}}, 30^{\mathrm{a}}, 35^{\mathrm{a}}, 40^{\mathrm{a}}, 50^{\mathrm{a}}, 60^{\mathrm{a}}, 70^{\mathrm{a}}, 80^{\mathrm{a}}, 90^{\mathrm{a}}$ e $100^{\mathrm{a}}$ espira da bobina, tendo a cabeça como referência. Cada espira foi cortada em 06 pedaços de aproximadamente 500 $\mathrm{mm}$ gerando 102 amostras que foram submetidas ao ensaio de tração e análise metalográfica. Os resultados do limite de escoamento obtidos nos ensaios de tração são mostrados no gráfico a seguir na base 100 .

Figura 4 - Resultados do Limite de Escoamento - Base 100

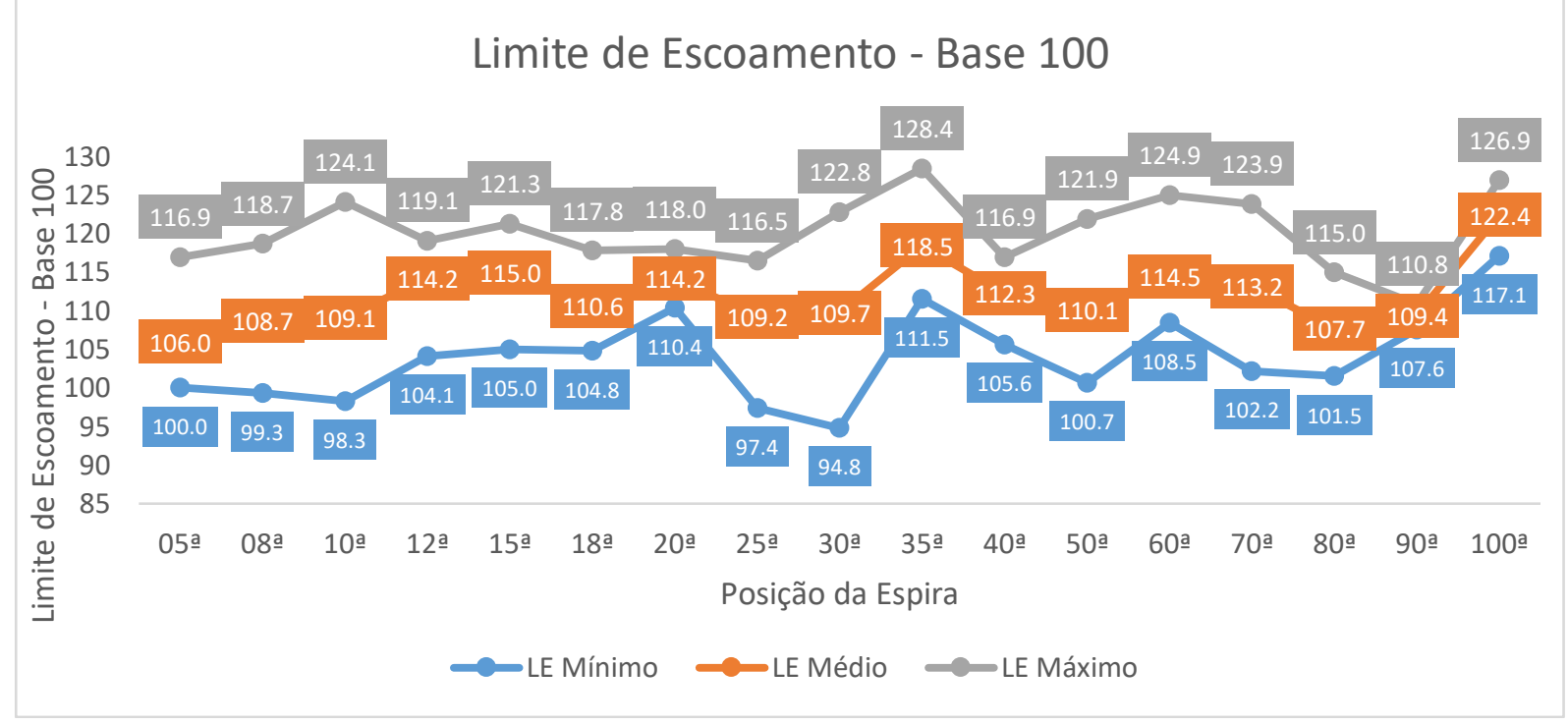

Foi observada uma grande variação nos resultados ao longo das espiras e bobinas. A partir da análise dos resultados obtidos e dos dados de processo, foram consideradas as variáveis que poderiam ter maior influência na média e desvio padrão do limite de escoamento, tais como:

- Distribuição da vazão nas caixas d'água;

- Tempo de ciclo do Formador de Espiras (Wobble) e;

- Ventilação do Stelmor. 
Com as variáveis definidas, foi elaborado um planejamento de experimentos (DOE) a fim de encontrar a melhor combinação entre as mesmas para obter um limite de escoamento acima do mínimo da norma e com menor desvio padrão. O DOE foi realizado conforme tabela abaixo, onde no primeiro experimento todas as variáveis ficaram com os valores padrões.

Tabela 2 - Planejamento de experimentos

\begin{tabular}{|c|c|c|c|c|c|c|}
\hline $\mathrm{C} 1$ & $\mathrm{C} 2$ & $\mathrm{C} 3$ & $\mathrm{C} 4$ & C5-T & C6-T & C7-T \\
\hline StdOrder & RunOrder & CenterPt & Blocks & Vazão Caixas D água & Tempo de Ciclo Wobble (s) & Ventilação do Stelmor (\%) \\
\hline 6 & 1 & 1 & 1 & Alta & 1 & 100 \\
\hline 2 & 2 & 1 & 1 & Alta & 1 & 10 \\
\hline 8 & 3 & 1 & 1 & Alta & 5 & 100 \\
\hline 4 & 4 & 1 & 1 & Alta & 5 & 10 \\
\hline 3 & 5 & 1 & 1 & Baixa & 5 & 10 \\
\hline 7 & 6 & 1 & 1 & Baixa & 5 & 100 \\
\hline 5 & 7 & 1 & 1 & Baixa & 1 & 100 \\
\hline 1 & 8 & 1 & 1 & Baixa & 1 & 10 \\
\hline
\end{tabular}

Em cada um dos 08 experimentos foi produzida 01 bobina e foram retiradas as 5a $30^{a}, 50^{a}$ e $90^{a}$ espiras. As espiras foram cortadas em 04 pedaços para serem submetidas ao ensaio de tração e metalografia.

\section{RESULTADOS E DISCUSSÃO}

A Figura 5 mostra o gráfico Boxplot de todos os experimentos onde destacase que os experimentos 5 e 8 apresentaram melhor média e menor desvio padrão quando comparado ao experimento 1 (padrão).

Figura 5 - Boxplot com os resultados dos experimentos (DOE)

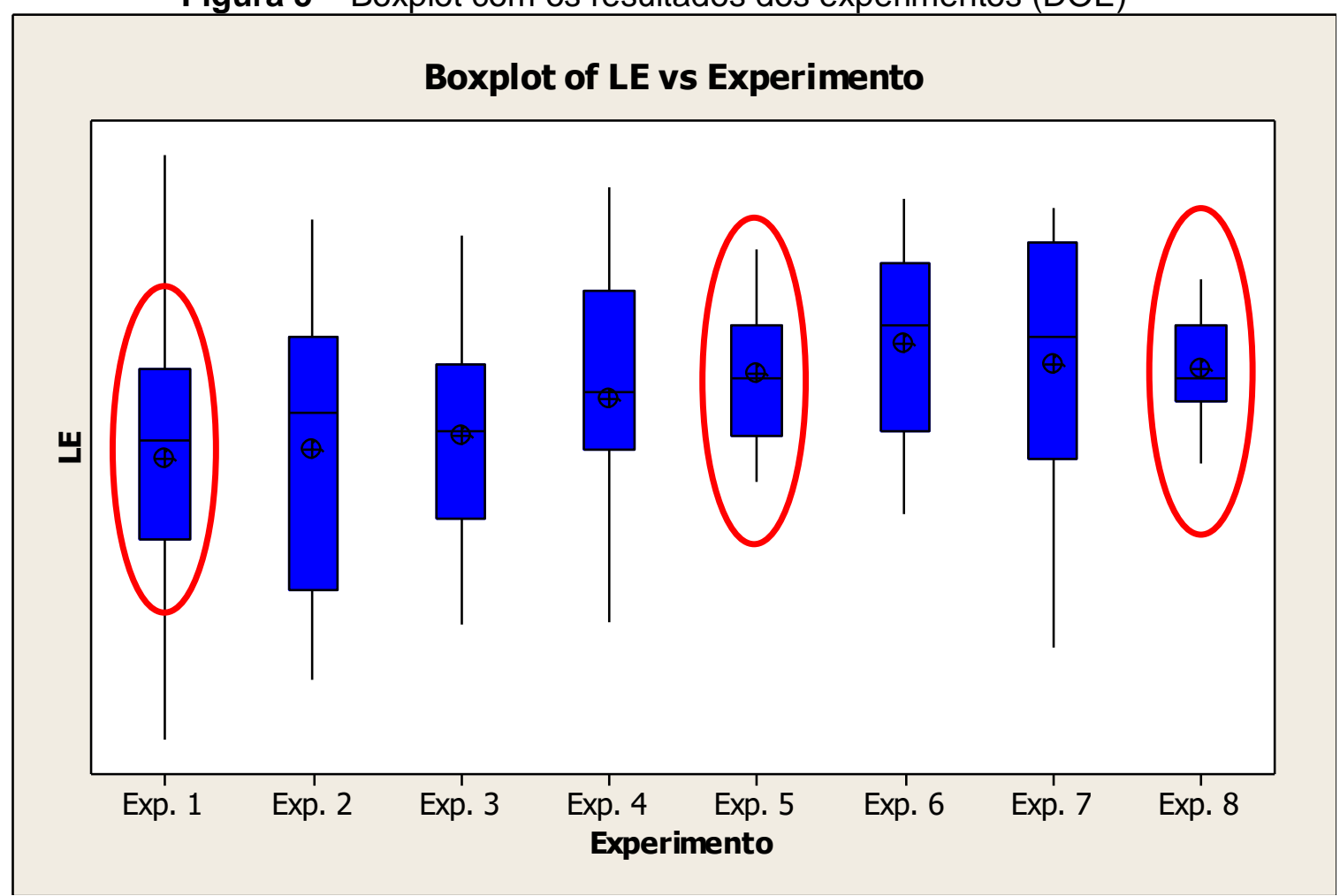


Tabela 3 - Resultados do limite de escoamento dos experimentos

\begin{tabular}{|l|c|c|}
\hline \multicolumn{2}{|c|}{$\begin{array}{c}\text { Limite de Escoamento - Base } \\
\text { Experimento }\end{array}$} & $\begin{array}{c}\mathbf{1 0 0} \\
\text { Desvio Padrão }\end{array}$ \\
\hline Experimento 1 & 107,8 & 11,1 \\
\hline Experimento 2 & 108,4 & 10,7 \\
\hline Experimento 3 & 109,6 & 7,8 \\
\hline Experimento 4 & 112,4 & 9,2 \\
\hline Experimento 5 & 114,2 & 5,2 \\
\hline Experimento 6 & 116,6 & 7,4 \\
\hline Experimento 7 & 115,0 & 9,9 \\
\hline Experimento 8 & 114,6 & 4,2 \\
\hline
\end{tabular}

Este fato pode ser explicado em função dois experimentos 5 e 8 terem trabalhado com menor quantidade de água na zona 2 das Caixas d'água. Além disso, a ventilação utilizada nestes experimentos foi a mínima, justificando o baixo desvio padrão pois não houve a influência do ar forçado no resfriamento as espiras no Stelmor.

\section{CONCLUSÃo}

Em função dos resultados obtidos nos experimentos, nota-se que ao reduzir a vazão da zona 2, com a manutenção da mesma temperatura no Formador de Espiras, foi possível obter um aumento do limite de escoamento do material de estudo. Este parâmetro somado à ventilação do Stelmor no valor mínimo e, com a maior distribuição das espiras, permitiram a redução do desvio padrão do limite de escoamento.

\section{CONCLUSÃO}

1 Das S., Mathur J., Bhattacharyya T., Bhattacharyya S., Failure Analysis Of Re-Bars During Bending Operations, Case Studies In Engineering Failure Analysis 2 (2014) 5153,2014

2 Official publications of the European communities, Quenching And Self-Tempering (QST) Of Beams In The Rolling Heat, 1992

3 Viventsov S., Kaskin B. K, Vdovin S. V, Serdyukov O. V. E Kutuzov V. V, Introduction Of A New Bar Rolling Mill 1, Steel In Translation Vol. 40 No. 62010

4 Copyright (C) Morgan Construction Company Manual Morgan Votorantim; Water Boxes Processing; Resende, 2009.

5 Copyright @ Morgan Construction Company Manual Morgan Votorantim; Water Boxes Processing; Resende, 2009.

6 Zakya A.I, El-Morsyb A., El-Bitar. T, Effect Of Different Cooling Rates On Thermo Mechanically Processed High-Strength Rebar Steel, Journal Of Materials Processing Technology $213818-825,2013$ 